\title{
Empowerment bei Älteren
}

\section{Empowerment for the Elderly}

Autoren

Institute

\section{U. Walter ${ }^{1}$, N. Schneider ${ }^{2}$, M. Plaumann}

${ }^{1}$ Prävention und Rehabilitation in der System- und Versorgungsforschung - Stiftungslehrstuhl am

${ }^{2}$ Institut für Epidemiologie, Sozialmedizin und Gesundheitssystemforschung, Medizinische Hochschule Hannover

\section{Schlüsselwörter \\ - Empowerment \\ - Gesundheitskompetenz \\ - Ältere \\ - Versorgung \\ - Arbeitnehmer}

\section{Key words \\ - empowerment \\ - health literacy \\ - elderly \\ - health care \\ - employees}

Bibliografie

DOI $10.1055 / \mathrm{s}-0028-1103270$

Gesundheitswesen 2008;

70: 730-735

(c) Georg Thieme Verlag KG

Stuttgart · New York

ISSN 0941-3790

Korrespondenzadresse

\section{Prof. Dr. U. Walter}

Prävention und Rehabilitation in der System- und Versorgungs-

forschung - Stiftungslehrstuhl Institut für Epidemiologie

Sozialmedizin und

Gesundheitssystemforschung

Medizinische Hochschule

Hannover

Carl-Neuberg-Straße 1

30625 Hannover

walter.ulla@mh-hannover.de

\section{Zusammenfassung}

$\checkmark$

Die aktive Gestaltung und Kontrolle der eigenen sozialen Lebenswelt wird als eine wesentliche Voraussetzung für körperliches und seelisches Wohlbefinden angesehen. Ein Konzept, welches Menschen dazu befähigen soll, ein eigenverantwortliches und selbstbestimmtes Leben zu führen, ist das Empowerment. Dieses sollte, u.a. aufgrund der demografischen Entwicklung, eine immer größere Bedeutung auch in der älteren Bevölkerungsgruppe erlangen. In unserer stark leistungsund arbeitsbezogenen Gesellschaft jedoch wird diese Gruppe von der Öffentlichkeit vor allem als Belastung betrachtet und ihr Potenzial, auch hinsichtlich eines förderlichen intergenerativen $\mathrm{Zu}-$ sammenlebens, gleichermaßen zu wenig berücksichtigt wie nicht hinreichend kommuniziert. Vorliegender Beitrag befasst sich mit Strategien des Empowerment bei Älteren. Verschiedene Ebenen werden erläutert und einzelne Ansätze vertieft betrachtet. Der Begriff Health Literacy wird in diesem Kontext als die Aneignung von Gesundheitskompetenz im Sinne von Verstehen gesundheitsbezogener Informationen bis hin zur Selbstgestaltung der eigenen Gesundheit verstanden. Verschiedene Studien, die Zusammenhänge zwischen Health Literacy und Health Outcomes bei Älteren untersuchen, werden aufgezeigt. Da die Förderung von Empowerment zudem in der Prävention und gesundheitsbezogenen Versorgung, z.B. in Form von „Komm“- und „Bring-Strukturen“ wiederzufinden ist, werden auch hierfür Beispiele genannt. Einen weiteren Fokus legt der Beitrag auf die Möglichkeit der Stärkung des Empowerment bei älteren Arbeitnehmern.

\section{Abstract \\ $\nabla$}

Active management and controlling of one's social setting is an essential basis for physical and psychological well-being. A concept which aims at building abilities for an individual responsible and autonomous life is empowerment. Due to the demographic development, this concept will become more important for the elderly. In our productive and work-life related society, this age group is often considered as a burden. Their age group-specific potential is not sufficiently recognised and utilised, e.g., regarding its possible supportive role in interactions between a community's generations. This article illustrates different levels and strategies of empowerment for the elderly and analyses some concepts. One of these concepts is health literacy, which is defined as gaining competence, e.g., for the understanding of information or for acting on health-related issues. Studies of association between health literacy and health outcomes in the elderly are represented. Additionally, the promotion of empowerment is part of the prevention and health care, e.g., in terms of "active management" and "passive management". A further focus is improving the empowerment in elderly employees. tive Gestaltung und Kontrolle der eigenen sozialen Lebenswelt angesehen. Empowerment hat zum Ziel, die Fähigkeiten von Menschen diesbezüglich zu entwickeln und zu optimieren so-
Als eine wesentliche Voraussetzung für körperliches und seelisches Wohlbefinden wird die ak- 
wie die Bedingungen zu schaffen, die ein eigenverantwortliches und selbstbestimmtes Leben ermöglichen [1,2]. Nach dem ursprünglich in der Sozialarbeit verankerten Konzept sollen u.a. diskriminierende Lebensbedingungen, wie z.B. negative Bewertungen und Stigmatisierungen infolge von Hilfebedürftigkeit, oder Behinderung durch gegenseitige Unterstützung und soziale Aktionen überwunden werden [3]. Empowerment kann aber auch darauf abzielen, den Betroffenen das Gefühl der Einflussnahme zurückzugeben und bestehende Hilflosigkeit zu überwinden [4]. Zur differenzierten und realistischen Wahrnehmung der Älteren sowie der Stärkung ihrer gesellschaftlichen und eigenen Achtung ist es - gerade in einer stark leistungs- und arbeitsbezogenen Gesellschaft - zudem wichtig, die ältere Bevölkerungsgruppe nicht nur als Belastung zu sehen, sondern auch ihre Potenziale zu erkennen und ihren Beitrag für ein förderliches intergeneratives Zusammenleben in der Öffentlichkeit sichtbarer hervorzuheben als es derzeit der Fall ist. Zu nennen sind an dieser Stelle insbesondere die Pflege von Angehörigen, die Betreuung von Kindern sowie ehrenamtliche Tätigkeiten in der Kommune.

Zugleich ist es vor allem in der vierten Lebensphase notwendig, den in den vorhergehenden Lebensphasen präventiv noch zu begegnenden biologischen, aber auch krankheitsbedingten Veränderungen Rechnung zu tragen [5]. So plädiert Morell [6], die sich mit dem Konzept des Empowerment bei Hochaltrigen auseinandersetzt, für ein alters-sensibleres Modell, das eine Co-Existenz zunehmender Vulnerabilität und „power“ erlaubt und damit sowohl gesundheitlichen Einschränkungen als auch Sterben und Tod die Stigmata nimmt.

Ausgehend von einer Übersicht zu individuellen sowie strukturellen Strategien zur Förderung von Empowerment bei Älteren werden im Folgenden einige Aspekte und Ansätze vertieft dargestellt.

\section{Ebenen des Empowerment bei Älteren \\ $\nabla$}

Strategien des Empowerment bei Älteren setzen auf verschiedenen Ebenen des Gesundheits-, Versorgungs- und Sozialsystems an, wobei sowohl organisationsbezogene als auch individuelle Methoden und Strategien unterschieden werden können [7-9]:

- Empowerment von Bürgern bzw. Patienten (patient empowerment) hat in den vergangenen Jahrzehnten unter der Prämisse, gesundheitsbezogene Outcomes zu verbessern, eine wesentliche Stärkung erfahren. Es kann bei den Betroffenen selber ansetzen, wie auch bei den Familien (family empowerment) und den Kommunen (community empowerment). Ziele sind die Förderung partnerschaftlicher Beziehungen zwischen Patienten und Leistungserbringern, die Förderung von sog. Self-Care-Strategien sowie die Verbesserung der Lebensqualität bei chronisch Kranken. Strategien sind in diesem Zusammenhang u.a. die Gesundheitsbildung, die Förderung individueller Fähigkeiten sowie die Entwicklung von Netzwerken.

Elemente des individuellen Empowerment sind ferner die Förderung der Teilnahme am Leben und die Gestaltung in der Gemeinde, die Verminderung von Hilflosigkeit, bis hin zur Förderung politischen Bewusstseins und der politischen Einflussnahme sowie die generelle Stärkung der Bürger/Patienten i.S. einer Verbesserung der Selbstwirksamkeit und des Selbstbewusstseins [3].
Ziele in der Kommune sind u.a. die Verringerung von sozialer Isolation und Entfremdung, die Ausschöpfung des gesundheitsbezogenen Potenzials der Älteren durch eine förderliche Gestaltung der strukturellen Rahmenbedingungen und die Förderung von Rechten und Mitsprache Älterer. Zur Umsetzung dieser Ziele ist seitens der Kommune nicht nur ein spezifisches Verständnis alter(ns)bezogener Bedarfe und Bedürfnisse erforderlich, sondern auch Kompetenz zur Zusammenbindung relevanter Akteure und die Verfügungsgewalt über zumindest grundlegende Ressourcen. Eine Befragung von 328 Städten, Gemeinden und Landkreisen in Deutschland [10] zeigt jedoch, dass in vielen Kommunen Gesundheitsförderung und Prävention als eigene Gestaltungsaufgaben bislang nur von mittlerer Bedeutung sind und eine konzeptionell orientierte Ausrichtung auf die Zielgruppe der Älteren bislang kaum stattgefunden hat. Allerdings sehen jeweils ca. $80 \%$ der befragten Kommunen eine stärkere Teilhabe älterer Menschen und die Erhöhung ihrer Selbstständigkeit als Ziele einer seniorenbezogenen Gesundheitsförderung und Prävention an. Ca. $60 \%$ benennen die Stärkung bürgerschaflichen Engagements Älterer als Ziel [10].

- Empowerment von Organisationen wie Leistungsanbietern und Akteuren im Gesundheitswesen sowie von wirtschaftlichen Unternehmen als Arbeitgeber für ältere Beschäftigte (provider bzw. organizational empowerment) umfasst einerseits die Professionellen, wie z.B. Ärzte und Pflegefachkräfte, mit dem Ziel, ihre Kompetenz, Arbeitsplatzzufriedenheit und Management-Fähigkeiten sowohl für ihre eigene Gesundheit als auch für die Stärkung des Patientenempowerment zu fördern. Zum anderen bezieht sich Empowerment hier auf gesundheitsbezogene Organisationen in der Absicht, die Versorgungsqualität zu erhöhen. Ähnliche Ziele und Ansätze können für Betriebe definiert werden mit der Absicht, das Empowerment ihrer Mitarbeiter zu stärken (s.u.).

- Gesellschaftliches Empowerment (societal empowerment) bezieht sich auf Teilpopulationen, die z. B. aufgrund von sozialer Diskriminierung von Entscheidungsprozessen ausgeschlossen worden sind. Ziel ist es, soziale, ökonomische oder politische Inbalancen zu verringern. Strategien sind mediale Informationen zur Beeinflussung der öffentlichen Wahrnehmung und Diskussion, die Förderung von Rechten sowie die Verabschiedung entsprechender Gesetze. Aufgrund der Bedeutung von sozialen Determinanten für den Gesundheitszustand kann hierdurch auch ein Beitrag für die Gesundheitsförderung geleistet werden.

In diesem Zusammenhang müssen auch Altersbilder, die sich im Wechselspiel zwischen Individuum und Gesellschaft herausbilden, betrachtet werden. Einerseits prägen alte Menschen Altersbilder durch ihr Handeln. Andererseits beeinflussen Altersbilder auf gesellschaftlicher und individueller Ebene die Wahrnehmung und Beurteilung von älteren Menschen sowie die Nutzung von Potenzialen und Kompetenzen für ein selbstbestimmtes und aktives, gesundes Altern, verbunden mit Erwartungen an den eigenen Alterungsprozess. Nicht zuletzt beeinflusst sowohl die Wahrnehmung der Älteren durch Dritte (Fremdbild) als auch die Selbstwahrnehmung der Älteren (Selbstbild) Wertschätzung, gesellschaftliche Akzeptanz sowie Gestaltung des Alterns [11]. Sollen ältere Menschen in einer Gesellschaft sozial, kulturell und politisch aktiv teilhaben, ist ein differenziertes, die Unterschiede in den körperlichen, geistigen und sozialen Ressourcen berücksichtigendes, Altersbild erforderlich [12]. 
Bedeutung von „Health Literacy“ (Gesundheitskompetenz) für die Gesundheit von Älteren

$\nabla$

Selbstbestimmung im Alter und Selbstgestaltung der Gesundheit erfordern ein hinreichendes Wissen um gesundheitsbezogene Aspekte, ein Verständnis für gesundheitsbezogene Informationen, eine gute Kenntnis der Unterstützungsangebote [13] sowie die Möglichkeiten ihrer Nutzung. Eine wesentliche Voraussetzung ist die Health Literacy (Gesundheitskompetenz) $[14,15]$. Diese umfasst die Lesefähigkeit von Gesundheitsinformationen sowie die Kompetenzen, diese zu verstehen, zu nutzen und daraus hervorgehende Gesundheitsleistungen in Anspruch zu nehmen sowie angemessene Entscheidungen zur Förderung und zum Erhalt der eigenen Gesundheit treffen zu können. Damit entspricht Health Literacy dem Verständnis der Ottawa Charta [16] und ist Teil des (individuellen) Empowerment. Als wesentliches Konzept und eine wichtige Determinante für Gesundheit hat Health Literacy im vergangenen Jahrzehnt in der Gesundheitsförderung und Prävention, aber auch in der Versorgung chronisch Kranker, eine zunehmende Bedeutung erlangt.

Health Literacy gilt als ein wesentlicher Prädiktor für die Gesundheit $[17,18]$. Unzureichende Health Literacy ist mit einem schlechten Gesundheitszustand, einer hohen Inanspruchnahme medizinischer Versorgung und schlechten funktionalen sowie klinischen Outcomes [19] assoziiert. Zu den Bevölkerungsgruppen, die gehäuft eine niedrige Gesundheitskompetenz (Health Literacy) aufweisen, zählen nach US-amerikanischen Studien sowie systematischen Reviews neben Personen mit einem geringeren sozio-ökonomischen Status bzw. geringerer Bildung und Migranten auch Ältere [20-22].

In einer Befragung von ca. 3000 neu eingeschriebenen, über 65 Jahre alten Patienten der amerikanischen Krankenversicherung Medicare wurden Gesundheitsverhalten, Gesundheit sowie Morbidität und Mortalität in Beziehung zu Health Literacy gesetzt. Ein Drittel der Befragten wies dabei eine geringe bzw. unzulängliche Health Literacy auf, gemessen an der Auffassungsfähigkeit von Texten und quantitativen Informationen. Nach dieser Studie ist eine unzureichende Gesundheitskompetenz ein Prädiktor für die Gesamt- sowie kardiovaskuläre Mortalität [18]. Ähnliche Ergebnisse zeigt eine prospektive Kohortenstudie bei 76- bis 81-Jährigen, wonach eine geringe Health Literacy mit einer zweifach erhöhten vorzeitigen Mortalität assoziiert ist [23]. Allerdings gaben Ältere mit geringer Health Literacy (anders als erwartet) häufiger an, niemals geraucht sowie Alkohol getrunken zu haben und führten häufiger einen bewegungsarmen Lebensstil. Eine unzureichende Health Literacy erwies sich jedoch nicht als unabhängiger Prädiktor für Rauchverhalten, Alkoholkonsum, geringe körperliche Aktivität und Übergewicht bzw. Adipositas [24]. Die Autoren konstatieren, dass es nach ihren Ergebnissen eher unwahrscheinlich ist, dass geringe Health Literacy das Gesundheitsoutcome direkt über das Gesundheitsverhalten beeinflusst. Möglicherweise spielen auch Faktoren wie das Wissen um chronische Krankheiten, Fähigkeiten zum Selbstmanagement sowie eine angemessene und zeitnahe Nutzung präventiver Maßnahmen eine Rolle.

Neben einer stärker eingeschränkten körperlichen Gesundheit weisen Ältere mit einer niedrigeren Health Literacy eine reduzierte psychische Gesundheit auf $[25,26]$. In einer Studie mit über 1000 Älteren unterschiedlicher Ethnien erwies sich das Ausmaß an Health Literacy aufgrund seiner vermutlich sensitiveren Reflexion bildungsbezogener Erfahrungen als besserer Prädiktor bezüglich einer Abnahme des Gedächtnisses, der Füh- rungsfunktion und Sprachfähigkeiten als die bloße Zählung der Bildungsjahre, die bislang als Proxy für kognitive Reserven angesehen worden sind. Manly et al. [27] schlussfolgern, dass Health Literacy als Mediator der Interaktionen zwischen biologischen und umweltbedingten Faktoren in Bezug auf die Verminderung kognitiver Fähigkeiten angesehen werden sollte. Zukünftige Forschung sollte sich daher durch ein vertieftes Verständnis für die Zusammenhänge zwischen Health Literacy und Health Outcome auszeichnen.

Durch die Verbesserung von Gesundheitswissen, Verstehen und Handlungsfähigkeit kann das Niveau der Health Literacy eines Menschen erhöht werden [28]. Nutbeam [14] weist auf die Notwendigkeit hin, die Kommunikation stärker auf die Zielgruppe anzupassen, wobei soziale und umfeldbezogene Einflüsse auf die Lebensweise einzubeziehen sind. Fachlich qualifizierte, die Motivation und das Lebensumfeld der Zielgruppe berücksichtigende, gesundheitsbezogene Informationen in leicht verständlicher Sprache müssen je nach Zielpopulation durch individuelle Beratung und weitere Strategien ergänzt werden.

\section{Ansätze zum Empowerment von Älteren im Rahmen der Prävention und gesundheitsbezogenen Versorgung \\ $\nabla$}

Der Ausbau der Bürger- und Patienteninformation und -beratung gilt als ein wichtiger Schritt zur Stärkung von Health Literacy [29] und damit von Empowerment. Hierzu wurde in Deutschland die Förderung unabhängiger Patientenberatungsstellen durch die Krankenkassen sozialgesetzlich verankert ( $\S 65 b$ Sozialgesetzbuch V). Diese Einrichtungen halten als „Komm-Strukturen“ ein Beratungsangebot für Bürgerinnen und Bürger zu gesundheitsbezogenen Themen vor. Erste Evaluationen von Schaeffer et al. [29] zeigen, dass die Beratungsstellen eher von jüngeren Personen genutzt werden, während ältere Menschen als eigentliche Hauptnutzer von Gesundheitsleistungen offenbar schlechter erreicht werden (weniger als ein Fünftel der schriftlich Ratsuchenden sind älter als 60 Jahre).

Ein weiteres Beispiel für „Komm-Strukturen“ sind die MiniMedSchools in den USA [30], nach deren Vorbild mittlerweile auch in Europa und Deutschland „Patientenuniversitäten“ entstanden sind. In unterschiedlichen Modulen richtet sich z. B. die Patientenuniversität an der Medizinischen Hochschule Hannover an gesunde Bürgerinnen und Bürger wie an erkrankte Personen. Vermittelt wird Basiswissen aus der Humanmedizin in laiengerechter Form und Sprache. Daneben soll auch die Systemkompetenz der Teilnehmer gestärkt werden, in dem u.a. aufgezeigt wird, auf welche Weise seriöse, wissenschaftlich fundierte Gesundheitsinformationen im Internet gefunden werden können. Nach ersten Evaluationen der Teilnehmerstruktur liegt das Durchschnittsalter bei 60 Jahren; überwiegend wird das Angebot von Frauen und Menschen mit hohem Bildungsniveau genutzt [31].

Deutlich wird, dass zur Förderung von Empowerment bei Älteren spezifische Ansätze erforderlich sind. Ein Beispiel hierfür ist das Hamburger Programm „Aktive Gesundheitsförderung im Alter“. Verschiedene Ansätze der Gesundheitsförderung und Primärprävention werden miteinander kombiniert, um die Eigenverantwortung von älteren Menschen in Kleingruppenarbeit zu stärken und durch professionelle Gesundheitsberater die Kompetenz für die selbstständige Umsetzung gesundheitsfördernder Maßnahmen zu vermitteln. Als „Komm-Struktur“ spricht das 
Angebot in erster Linie mobile, motivierte und kontaktfreudige Personen an [32].

Ein Beispiel für eine „Bring-Struktur“ hingegen ist ein Projekt, das im Rahmen des „Active Ageing“-Programms der Weltgesundheitsorganisation in der nordrhein-westfälischen Kleinstadt Radevormwald durchgeführt wurde. Zielgruppe waren Personen zwischen 55 und 80 Jahren in einer kritischen Lebensphase (Verlust des Partners oder Ausscheiden aus dem Berufsleben). Die Teilnehmer wurden über zwei Jahre zu Hause von geschulten Beratern (Sozialarbeiter, Pflegefachkräfte, Sozialpädagogen) aufgesucht, um individuelle Möglichkeiten für Aktivitäten zu erarbeiten. Gezeigt werden konnte unter anderem, dass sich bei Teilnehmern mit Verlust des Lebenspartners innerhalb der vorangegangenen fünf Jahre eine Intensivierung sozialer Aktivitäten (z.B. vermehrte Teilnahme an Gruppenaktivitäten) sehr positiv auf die Lebensqualität auswirkte. Die Ergebnisse dieses Projekts unterstreichen auch die Notwendigkeit einer Risikogruppenstrategie, denn identische Interventionsmaßnahmen führten beispielsweise bei älteren Menschen in einer Übergangsphase wie dem Ausscheiden aus dem Erwerbsleben nicht zu verbesserter Lebensqualität [33].

Neben der Identifikation und Nutzung wirksamer Zugangsstrukturen ist zur Steigerung des Empowerments eine zielgruppenorientierte Ansprache erforderlich. Hierzu liegen für die Bevölkerungsgruppe der Älteren bislang nur wenige Erfahrungen vor. Im Rahmen eines eigenen, in Zusammenarbeit mit dem Institut für Allgemeinmedizin der Medizinischen Hochschule Hannover und der AOK Niedersachsen durchgeführten BMBF-geförderten Projektes wird derzeit untersucht, ob und inwieweit eine z.B. nach Alter und Geschlecht differenzierte Ansprache erforderlich ist, um Ältere für eine Maßnahme des präventiven Hausbesuchs zu gewinnen [34].

Ein wesentlicher Aspekt bei der Förderung der Selbstständigkeit im Alter ist die Vermeidung durch „Entmündigung“ infolge von Überbetreuung sowohl seitens der Professionellen als auch der Angehörigen. Rahmenbedingungen wie ökonomisch festgelegte Zeitbudgets, aber auch eine vermeintlich gut gemeinte Übernahme von Hilfeleistungen, fördern häufig die Unselbstständigkeit der Adressaten. Ein schon klassisches Trainingsprogramm für Pflegende der Arbeitsgruppe um Margret Baltes zeigt eindrucksvoll, wie die Selbstständigkeit von Demenzkranken durch eine erhöhte Sensibilisierung der Pflegekräfte für die Bedürfnisse und Ressourcen der Pflegebedürftigen sowie durch eine Veränderung der Arbeitsroutinen gefördert werden kann [35]. Nomura et al. [36] konnten durch kognitive Rehabilitationsmaßnahmen (Wiedererarbeiten verlernter oder vergessener Stärken) eine Steigerung des Selbstwertgefühls und des Vertrauens in die eigenen Fähigkeiten bei Demenzkranken in frühen Stadien erreichen.

Zielgruppen des Empowerment-Programms von Nomura et al. [36] waren neben den Demenzkranken auch ihre (pflegenden) Angehörigen. Pflegende Angehörige, oft die Lebenspartner der Demenzkranken, nehmen ihre eigene Gesundheit als erheblich schlechter wahr, verglichen mit den altersspezifischen Normwerten der Allgemeinbevölkerung. Darüber hinaus klagen sie weitaus häufiger über eigene körperliche und seelische Beschwerden wie Erschöpfung, Herz-Kreislauf- und Magenprobleme als Pflegende von kognitiv nicht wesentlich beeinträchtigten Patienten. In der Folge kommt es bei den Pflegenden gehäuft zu depressiven Verstimmungen, psychosomatischen Störungen, Erkrankungen des Bewegungsapparats oder zur Einnahme von Psychopharmaka. Oft ist die Überlastung der Angehörigen der Grund für eine Heimunterbringung des Erkrankten [37]. Gezielte pflegetechnische sowie psychosoziale Unterstützung einschließlich konkreter Entlastungsmaßnahmen und Hilfestellungen im Umgang mit den erforderlichen administrativen Regelungen können hier dem Laien bei der Pflege seiner Angehörigen und dem Erhalt seiner eigenen Gesundheit helfen. Um Empowerment bei pflegenden Angehörigen zu fördern, sollten entsprechende Maßnahmen möglichst frühzeitig in den Pflegeprozess integriert werden, so z.B. Beratung und Schulung in Stressbewältigung und Pflegetechniken $[36,38]$.

Barrieren für Empowerment bei Älteren sind aufseiten der Gesundheitsprofessionen Qualifikationsdefizite (z.B. Wissen zu Prävention und Health Literacy), Einstellungen (z.B. Altersbilder) und Rollenverständnis (z.B. berufliche Identität) [39]. Wichtig ist eine Qualifizierung der Professionellen hinsichtlich der Wirksamkeit gezielter, auch präventiver und gesundheitsfördernder Maßnahmen im Alter und ihre Umsetzung in der Praxis. Mit der kürzlich erfolgten Integration spezifischer alterssowie präventions- und gesundheitsförderungsbezogener Inhalte z.B. in die ärztliche und pflegerische Ausbildung wird versucht, diesem Defizit zu begegnen [5].

\section{Empowerment bei älteren Arbeitnehmern stärken: Arbeitsplatz-, individualressourcen- und gesellschaftsbezogene Ansätze $\checkmark$}

In Zukunft wird das durchschnittliche Alter der arbeitenden Bevölkerung aufgrund der demografischen Entwicklung und der Anhebung des Renteneintrittalters steigen. So ist hinsichtlich der Alterung innerhalb der erwerbstätigen Bevölkerung zu erwarten, dass der Anteil der mittleren Altersgruppe (30-49 Jahre) von derzeit $50 \%$ auf ca. $43 \%$ im Jahr 2050 absinken wird. Im Gegenzug erhöht sich der Anteil der älteren Altersgruppe (5064 Jahre) von $30 \%$ auf ca. $40 \%$. Somit sind diese beiden Altersgruppen im Erwerbsleben nahezu gleich stark vertreten [40]. Diese Berechnungen weisen u.a. auf die zunehmende Bedeutung der älteren Erwerbstätigen als Zielgruppe in der Arbeitswelt hin. Allerdings zeigt die Kultur der Frühverrentung, die zumindest in einigen Branchen - immer noch von Unternehmen gefördert wird, dass diese Bedeutung bislang nicht gänzlich erkannt wurde. Die zukünftige Herausforderung besteht von daher vor allem darin, die Einbindung der älteren Arbeitnehmer in den Arbeitsmarkt beizubehalten und zu fördern. Dieses Ziel kann jedoch nur dann erreicht werden, wenn ältere Arbeitnehmer die Möglichkeit haben, sich Fähigkeiten anzueignen, die es ihnen erlauben, dem Arbeitsmarkt bis ins höhere Alter zur Verfügung zu stehen. Ansätze des Umdenkens zeigen sich in den letzten Jahren auch innerhalb der Europäischen Union, die sich im März 2001 auf einer Tagung des Europäischen Rates in Stockholm u.a. das Ziel gesetzt hat, die Steigerung der durchschnittlichen EU-Beschäftigungsquote für ältere Männer und Frauen (zwischen 55 und 64 Jahren) bis 2010 auf 50\% zu steigern [41]. Deutschland hat diesen Vorsatz 2007 mit einer Erwerbsquote älterer Erwerbstätiger in Höhe von 51,5\% erreicht (Frauen: 43,6\%, Männer: 59,7\%) [42].

Empfehlungen, die dazu beitragen können, dass ältere Erwerbstätige länger gesund und motiviert arbeiten, können in drei Bereiche aufgeteilt werden [43]. Während der erste Bereich Empfehlungen zu arbeitsplatzbezogenen Rahmenbedingungen aufzeigt, fokussiert sich der zweite auf persönliche Ressourcen und der dritte auf gesellschaftliche Einstellungen. 
Tab. 1 Empfehlungen zum Empowerment älterer Arbeitnehmer (übersetzt und modifiziert nach [43]).

\begin{tabular}{|c|c|c|}
\hline Arbeitsplatzbezogene Empfehlungen & Individualressourcenbezogene Empfehlungen & $\begin{array}{l}\text { Gesellschaftsbezogene } \\
\text { Empfehlungen }\end{array}$ \\
\hline $\begin{array}{l}\text { Psychologisch wirksame Rahmenbedingungen } \\
\text { bei der Arbeit von älteren Erwerbstätigen sollten } \\
\text { dahingehend gestaltet werden, dass ihre Stärken un- } \\
\text { terstützt und entsprechend genutzt werden können. } \\
\text { Ältere Erwerbstätige benötigen zudem inspirierende } \\
\text { und herausfordernde Arbeit, durch die sie sich neue } \\
\text { Fähigkeiten und Fertigkeiten aneignen können. }\end{array}$ & $\begin{array}{l}\text { Gesundheitsförderliche Maßnahmen hinsichtlich älterer } \\
\text { Erwerbstätiger sollten verstärkt werden - die eigene Verantwor- } \\
\text { tung für die Lebensgewohnheiten ist von großer Bedeutung. }\end{array}$ & $\begin{array}{l}\text { Jeder sollte das Recht auf } \\
\text { lebenslanges Lernen haben. }\end{array}$ \\
\hline \multirow[t]{3}{*}{$\begin{array}{l}\text { Die Möglichkeiten älterer Erwerbstätiger, ihre eigene } \\
\text { Arbeit zu planen und zu regeln, sollten verbessert } \\
\text { werden. }\end{array}$} & $\begin{array}{l}\text { Funktionelle Leistungsfähigkeit sollte unterstützt und multidi- } \\
\text { mensional entstehen können - Leistungsfähigkeit hinsichtlich } \\
\text { physiologischer, mentaler und sozialer Funktionen ist elemen- } \\
\text { tare Voraussetzung für ein gutes Arbeitsleben, eine hohe Le- } \\
\text { bensqualität während der Arbeit sowie für erfolgreiches Altern. }\end{array}$ & $\begin{array}{l}\text { Die Neuausrichtung des Ar- } \\
\text { beitslebens in eine das höhere } \\
\text { Alter befürwortende Richtung } \\
\text { sollte ein gemeinsames Ziel für } \\
\text { Menschen aller Altersgruppen } \\
\text { sein. }\end{array}$ \\
\hline & $\begin{array}{l}\text { Älteren Erwerbstätigen sollte die Möglichkeit des lebenslangen } \\
\text { Lernens angeboten werden, und ihre berufliche Kompetenz } \\
\text { sollte sich durch die Anwendung angemessener Lernprozesse } \\
\text { weiterentwickeln. }\end{array}$ & $\begin{array}{l}\text { Der Übergang in die Beren- } \\
\text { tung sollte ein Prozess sein, } \\
\text { auf den sich die Angestellten } \\
\text { in ihren letzten Arbeitsjahren } \\
\text { vorbereiten können. }\end{array}$ \\
\hline & $\begin{array}{l}\text { Personen mit reduzierter Arbeitsfähigkeit sind ebenfalls ein } \\
\text { wichtiger Teil der gesamten Arbeitskraft - ihre verbleibende } \\
\text { Arbeitsfähigkeit sollte besser im Arbeitsleben genutzt werden. }\end{array}$ & \\
\hline
\end{tabular}

Alle drei Bereiche enthalten Elemente der Eigenverantwortung und Selbstbestimmung und somit Inhalte des Empowerment-Konzepts ( $\bullet$ Tab. 1).

Zur Förderung ihres Empowerment sollten ältere Arbeitnehmer für Tätigkeiten zuständig bzw. verantwortlich sein, die als inspirierend und herausfordernd empfunden werden. Unter psychologisch geeigneten Rahmenbedingungen können ältere Arbeitnehmer sich neue Fähigkeiten und Fertigkeiten aneignen und ihre Arbeit als Bereicherung sehen. Sie sollen in die Lage versetzt werden, ihre Arbeit inhaltlich eigenständig zu planen und ihre Arbeitszeit individuell zu gestalten.

Mit dem Ziel der eigenverantwortlichen Gestaltung ihrer Lebensgewohnheiten, sollten gesundheitsförderliche Maßnahmen verstärkt werden. Die Aufrechterhaltung physischer und psychischer Leistungsfähigkeit ist Voraussetzung eines als erfolgreich erlebten Arbeitslebens und Alterns. Ist die Leistungsfähigkeit nicht mehr vollständig gegeben, sollten die übrig gebliebenen Fertigkeiten in den Arbeitsprozess optimal integriert werden.

Neben dem Recht auf lebenslanges Lernen besteht auch die Verpflichtung lebenslanges Lernen zu ermöglichen. Ein gesamtgesellschaftliches Ziel sollte zudem eine positiv konnotierte Wertschätzung älterer Menschen im Arbeitsleben sein. Es sollte den älteren Arbeitnehmern ermöglicht werden, sich auf den Übergang in die Berentung vorbereiten zu können.

Wie eine bundesweite Umfrage der Bertelsmann Stiftung zur „Beschäftigungsfähigkeit und Aktivität bis ins Alter“ zeigt, werden einige dieser Ansatzpunkte auch vonseiten der Arbeitnehmer gewünscht, die sich derzeit mitten in der Erwerbsphase befinden [12]. Ungefähr drei Viertel der befragten 35- bis 55jährigen Erwerbstätigen möchten bis zum Erreichen ihres Renteneintrittsalters beruflich aktiv bleiben. Eine zentrale Voraussetzung dafür ist u.a. die lebenslange Erhaltung der Beschäftigungs- und Lernfähigkeit. Dabei sehen sich 94\% der Erwerbstätigen selbst am stärksten in der Verantwortung, wenn es um den Erhalt ihrer Beschäftigungsfähigkeit geht, und ca. drei Viertel der Erwerbstätigen wollen diese durch kontinuierliche Weiter- bildung sichern und stärken. Den direkten Vorgesetzten und der Unternehmensleitung wird dabei eine Mitverantwortung zugesprochen. Die Hälfte der befragten Erwerbstätigen sehen Bildungseinrichtungen und Anbieter von Fort- und Weiterbildungen ebenfalls mitverantwortlich dafür, dass sie in die Lage versetzt werden, den gegenwärtigen und zukünftigen Anforderungen im Erwerbsleben standzuhalten. 70\% der Befragten wünschen sich zudem mehr Anerkennung ihrer Arbeitsleistungen durch ihre Vorgesetzten und sehen darin eine hauptsächliche Motivation, ihrer derzeitigen beruflichen Tätigkeit bis zum 65. Lebensjahr nachzugehen. Die Sicherung ihrer Beschäftigungsfähigkeit durch den Arbeitgeber in Form von angemessenen Herausforderungen und anspruchsvolleren Tätigkeiten wünschen sich 86\% der Befragten.

\section{Fazit}

Konzepte von Empowerment bei Älteren im Rahmen der Prävention lassen sich in der internationalen Literatur ansatzweise finden. Vor dem Hintergrund des demografischen Wandels und der Notwendigkeit der Förderung der Selbstständigkeit im Alter steigt die Bedeutung der Umsetzung wirksamer Empowerment-Konzepte auf kommunaler Ebene auch für Akteure im Gesundheits- und Sozialwesen.

\section{Literatur}

1 Stark W. Empowerment. In: BzgA, Hrsg. Leitbegriffe der Gesundheitsförderung. Schwabenheim: Peter Sabo; 2003; 28-31

2 Brüggemann $S$, Irle $H$, Mai $H$ et al. Hrsg. Pschyrembel Sozialmedizin. Berlin: de Gruyter; 2007

3 Kam PK. Empowering elderly people: a community work approach. Community Dev J 1996; 31: 230-240

4 Muss A. Empowerment. Psych Pflege 2002; 8: 277-284

5 Walter U, Schneider $N$. Gesundheitsförderung und Prävention im Alter - Realität und professionelle Anforderung. In: Hensen G, Hensen P, Hrsg. Gesundheitswesen und Sozialstaat. Leverkusen: VS - Verlag für Sozialwissenschaften; 2008

6 Morell CM. Empowerment and long-living women: return to the rejected body. J Aging Stud 2003; 17: 69-85 
7 Loukanova SN, Bridges JFP. Empowerment in medicine: an analysis of publication trends 1980-2005. Cent Eur J Med 2008; 3 (1): 105-110

8 Wallerstein $N$. What is the evidence on the effectiveness of empowerment to improve health?. Copenhagen: WHO Regional Office for Europe Health Evidence Network report; 2006; http://www.euro.who. int/Document/E88086.pdf [20.4.2008].

9 Kar SB, Pascual CA, Chickering KL. Empowerment of women for health promotion: a meta-analysis. Soc Sci Med 1999; 49: 1431-1460

10 Hollbach-Grömig B, Seidel-Schulze A. Seniorenbezogene Gesundheitsförderung und Prävention auf kommunaler Ebene - eine Bestandsaufnahme. Köln: BZgA; 2007

11 Walter $U$, Flick $U$, Neuber A et al. Alt und gesund? Altersbilder und Präventionskonzepte in der ärztlichen und pflegerischen Praxis. Leverkusen: VS - Verlag für Sozialwissenschaften; 2006

12 Bertelsmann Stiftung. Alter neu denken. Empfehlungen der Expertenkommission „Ziele in der Altenpolitik“ zu gesellschaftlichen Altersbildern. Gütersloh: Bertelsmann Stiftung; 2006

13 Döhner H. Information und Beratung - Ein Beitrag zur Gesundheitsförderung und -versorgung. Z Gerontol Geriat 2001; 34 (1): 48-55

14 Nutbeam $D$. Health literacy as a public health goal: a challenge for contemporary health education and communication strategies into the 21st century. Health Promot Int 2000; 15 (3): 259-267

15 Bastian H, Kaiser T, Matschewsky S. Förderung allgemeiner Gesundheits- und Wissenschaftskenntnisse mittels Bürger- und Patienteninformation: Die Rolle des IQWiG. ZaeFQ 2005; 99 (6): 379-385

16 Kickbusch I. Health literacy: addressing the health and education divide. Health Promot Int 2001; 16 (3): 289-297

17 Ad Hoc Committee on Health Literacy for the Council on Scientific Affairs, American Medical Association. Health literacy: report of the Council on Scientific Affairs. JAMA 1999; 281 (6): 552-557

18 Baker DW, Wolf MS, Feinglass $J$ et al. Health literacy and mortality among elderly persons. Arch Intern Med 2007; 167 (14): 15031509

19 Wolff MS, Gazmarian JA, Baker DW. Health literacy and functional health status among older adults. Arch Intern Med 2005; 165 (17): 1946-1952

20 Nielsen-Bohlman L, Panzer AM, Kindig DA. Health literacy: a prescription to end confusion. Institute of Medicine. Washington DC: National Academies Press; 2004

21 Health literacy. A prescription to end confusion. Institute of Medicine. Washington DC: National Academies Press; 2004

22 Paaschke-Orlow MK, Parker RM, Gazmararian JA et al. The prevalence of limited health literacy. J Gen Intern Med 2004; 20: 175-184

23 Sudore RL, Yaffe K, Satterfield S et al. Limited literacy and mortality in the elderly. The health, aging and body composition study. J Gen Intern Med 2006; 21 (8): 806-812

24 Wolf MS, Gazmararian JA, Baker DW. Health literacy and health risk behaviors among older adults. Am J Prev Med 2007; 32 (1): 19-24

25 Wolf MS, Gazmararian JA, Baker DW. Health literacy and functional health status among older adults. Arch Intern Med 2005; 165 (17): 1946-1952

26 Howard DH, Sentell T, Gazmararian JA. Impact of health literacy on socioeconomic and racial differences in health in an elderly population. J Gen Intern Med 2006; 21 (8): 857-861
27 Manly JJ, Schupf N, Stern Y. Cognitive decline and literacy among ethnically diverse elders. J Geriatr Psychiatry Neurol 2005; 18: 213217

28 Osborne $H$. Health literacy from A to Z. practical ways to communicate your health message. Dudbury, Massachusetts: Jones and Bartlett Publischers; 2004

29 Schaeffer D, Dierks ML, Hurrelmann K et al. Evaluation der Modellprojekte zur unabhängigen Patientenberatung und Nutzerinformation. Bern, Göttingen, Toronto, Seattle: Hans Huber; 2005

30 Cohen I. How to start and conduct a MiniMed-School 2003; http:// www.uchsc.edu/minimed/minimed_howto.htm [19.11.2008]

31 Dierks $M L$, Seidel $G$, Lingner $H$ et al. Die Patientenuniversität an der Medizinischen Hochschule Hannover. Managed Care 2007; 7/8: 34-39

32 Dapp U, Anders J, Meier-Baumgartner $H$ et al. Geriatrische Gesundheitsförderung und Prävention für selbstständig lebende Senioren: Angebote und Zielgruppen. Z Gerontol Geriat 2007; 40: 226-240

33 Knesebeck $O$, David K, Bill $P$ et al. Aktives Altern und Lebensqualität - Evaluationsergebnisse eines WHO-Demonstrationsprojektes. Z Gerontol Geriat 2006; 39: 82-89

34 Theile G. Präventive Hausbesuche: ein Baustein in der ambulanten Versorgung älterer Menschen. Public Health Forum 2007; 15 (57): $22-24$

35 Neumann EM, Zank S, Tzschätzsch K et al. Selbstständigkeit im Alter. 2., korr. Aufl. Bern, Göttingen, Toronto, Seattle: Hans Huber; 1997

36 Nomura $M$, Makimoto $K$, Kato $M$ et al. Empowering the elderly with early dementia and family caregivers: A participatory action study. International Journal of Nursing Studies 2007, doi:10.1016/j.jinur stu.2007.09.009

37 Gesundheitsberichterstattung des Bundes. Gesundheit in Deutschland. Berlin; 2006

38 Walter U, Schneider N. Ansätze zur Gesundheitsförderung und Prävention. In: Ministerium für Arbeit, Gesundheit und Soziales des Landes Nordrhein-Westfalen, Hrsg. Gesundheitsberichte Spezial. Band 4: Gesundheit im Alter - demographische Grundlagen und präventive Ansätze. Düsseldorf; 2007

39 Scheaffer D, Schmidt-Kaehler S Hrsg. Lehrbuch Patientenberatung. Bern: Hans Huber; 2006

40 Bevölkerung Deutschlands bis 2050. In: Statistisches Bundesamt, Hrsg. 11. koordinierte Bevölkerungsvorausberechnung. Presseexemplar. Wiesbaden: Pressestelle; 2006

41 Europäischer Rat. Schlussfolgerungen des Vorsitzes. 23. und 24. März 2001. Stockholm: Europäischer Rat; 2001; http://europa.eu/european-council/index_de.htm [26.5.2008]

42 Beschäftigungsquote älterer Erwerbstätiger - insgesamt. Beschäftigungsquote älterer Erwerbstätiger - weiblich Beschäftigungsquote älterer Erwerbstätiger - männlich. Eurostat; 2008; http://epp.euro stat.ec.europa.eu [26.5.2008]

43 Ilmarinen J. Towards a better worklife. In: Finnish Institute of Occupational Health, Ministry of Social Affairs and Health, Ilmarinen Juhani, Hrsg. Towards a longer worklife! Ageing and the quality of worklife in the European Union. Helsinki: Finnish Institute of Occupational Health; 2005; 389-424 